\title{
Human nonverbal counting estimated by response production and verbal report
}

\author{
MICHAEL J. BOISVERT, BENJAMIN D. ABROMS, and WILLIAM A. ROBERTS \\ University of Western Ontario, London, Ontario, Canada
}

\begin{abstract}
In three experiments, people were shown sequential displays and were prevented from verbal counting by being required to perform other cognitive tasks. In Experiment 1, the subjects were shown three target (target $=8,16$, or 32) sequences of colored geometric shapes. On occasional question trials, the subjects were asked to estimate the target number after the final item in the sequence. On other test trials, items continued to appear beyond the target, and the subjects estimated the target manually by tapping a space bar. In Experiments 2 and 3, a matching-to-sample procedure required the subjects to estimate the same sequence of items (target $=8,11,14,17$, or 20 ) both verbally and manually. The results indicated that (1) manual and verbal estimates closely approximated target size in Experiments 1 and 2, (2) coefficients of variation were constant across target size, and (3) correlations between manual and verbal estimates were positive in Experiments 2 and 3. Requiring the subjects to perform a counting task during presentation of items led to underestimation of number in Experiment 3.
\end{abstract}

Although counting is typically thought of as being accomplished by the assignment of verbal number symbols to things counted, accumulating evidence suggests that counting also may take place nonverbally. The evidence appears in three different areas of research: experiments with animals, experiments with preverbal children, and most recently, experiments with adult humans instructed not to count verbally.

Animals as diverse as rodents, birds, and nonhuman primates have learned to discriminate between different numbers of stimuli presented either simultaneously or successively (see Roberts, 1998, 2002, for reviews). In other studies, rats were rewarded for making a fixed number of responses. Thus, the rats were required to make $N$ or more presses on a bar before executing a second operant response for reinforcement (Mechner, 1958; Platt \& Johnson, 1971). When $N$ varied from 4 to 24 , the average number of barpresses closely approximated $N$. In pigeon experiments, birds were presented with a key that flashed red for $200 \mathrm{msec}$ at either a fast rate $(2 / \mathrm{sec})$, a medium rate $(1 / \mathrm{sec})$, or a slow rate $(0.5 / \mathrm{sec})$, and the first peck made on the key after $N$ flashes produced reinforcement. On peak number test trials, the key continued to flash beyond $N$ flashes, and no reinforcer was delivered. The peak of the distribution of pecking responses made on test trials closely approximated $N$ flashes regardless of the rate at which the key flashed (Roberts \& Boisvert, 1998; Roberts,

This research was funded by a research grant from the Natural Sciences and Engineering Research Council of Canada to W.A.R. and by the Ontario Graduate Scholarship in Science and Technology and the Ontario Graduate Scholarship to M.J.B. Correspondence concerning this article should be addressed to M. J. Boisvert or W. A. Roberts, Department of Psychology, University of Western Ontario, London, ON, N6A 5C2 Canada (e-mail: mjboisve@uwo.ca or roberts@uwo.ca).
Coughlin, \& Roberts, 2000; Roberts, Roberts, \& Kit, 2002). In both the rat and the pigeon studies, approximate Gaussian distributions of response frequencies were found around the peak number of responses, and the width of these distributions was proportional to $N$. A proportional increase in response variability with an increase in the value being estimated is referred to as the scalar property and is commonly found in studies of animal timing and counting.

The fact that infants' looking time habituates with repeated observation of the same display and dishabituates when a novel display is shown has been used to study numerical abilities. Infants' looking time decreases when they are repeatedly shown displays containing a small fixed number of objects (two or three) but then rises when the number is changed by adding or subtracting an object (Starkey \& Cooper, 1980; Strauss \& Curtis, 1981; Van Loosbroek \& Smitsman, 1990). Other studies suggest that infants dishabituate looking time when number of sounds or number of sequential actions is changed (Starkey, Spelke, \& Gelman, 1990; Wynn, 1996) or when a display contains the incorrect number of objects after an object has been added to or subtracted from the display (Wynn, 1992). Wynn $(1995,1997)$ has argued from these findings that infants are born with the ability to represent different numbers of objects or events as different mental representations. Her position has been challenged by others who have suggested that infants' apparent numerical skills may arise from low-level perceptual processes, instead of numerical processes. Infants might be dishabituating to changes in number of objects or events either because they are attending to some correlated continuous variable, such as contour length (Clearfield \& Mix, 1999), or because they are representing a display as a set of object files (Feigenson, Carey, \& Hauser, 2002; Simon, 1997). Object file theory suggests that a small number of one to three 
objects or events are stored in memory as abstract symbols that have no numerical representation. When a new array is seen, its objects are mapped to the files on a one-to-one basis. If the array and files do not match up, the infant will show increased looking time. Since the infant's attention is held to be limited to about four items (Simon, 1997), dishabituation effects should be limited to four or fewer objects or events. Recent experiments by $\mathrm{Xu}$ and Spelke (2000) have shown, however, that 6-month-old infants can discriminate between displays containing 8 and 16 patterns, but not between displays containing 8 and 12 patterns. Tests of numerical discrimination and estimation with older children, 3-7 years old, have shown clear evidence of Weber's law and the scalar property. Displays containing a 2:3 ratio of patterns were harder to discriminate than those containing a 1:2 ratio (Huntley-Fenner \& Cannon, 2000), and the variability of the estimation of the number of patterns seen in a briefly shown array increased in proportion to the mean estimate (Huntley-Fenner, 2001).

Yet a third approach to nonverbal counting has been to use human subjects who have been instructed not to count. Whalen, Gallistel, and Gelman (1999) showed adults an Arabic numeral that varied randomly from 7 to 25 and asked the subjects to press a key as rapidly as possible until they felt they had reached the specified number. The subjects were instructed not to count their presses, and measurement of pressing rates suggested that the subjects pressed the key at a rate faster than they could count subvocally. The mean number of presses the subjects performed closely approximated the specified Arabic number over the full range of values for each subject tested. Evidence for scalar variability was found; coefficients of variation (COVs; standard deviation divided by the mean) remained invariant across numbers estimated. In a recent report, Cordes, Gelman, Gallistel, and Whalen (2001) replicated this procedure, with modification. One group of subjects was told to pronounce the word "the" each time the key was pressed, and two other groups were told to verbally count presses, one by individual integers and the other by tens. All three groups showed accurate increases in mean presses as the target number increased. A finding of major importance was that the COVs remained constant across mean number of presses in the "the" group but declined with number of presses in the two counting groups. Cordes et al. argued that these two patterns represent qualitatively different variability signatures. Requiring subjects to say "the" each time they pressed prevented subvocal counting and led to numerical estimation based on an accumulating magnitude that showed scalar variability. Subjects who were allowed to count made occasional errors by either omitting a number or repeating a number; variability in these counts should increase as the square root of the target number and, thus, lead to declining COVs as number increases.

Research in all three of the areas reviewed may be integrated within the framework of the dual-mode model of timing and counting introduced by Meck and Church
(1983) to account for the results of rat studies. When the proposed mechanism operated in the event mode, each time a stimulus was perceived, a switch would close and allow a fixed number of pulses to be transmitted from a pulse generator to an accumulator. The total pulses accumulated after a sequence of stimuli was stored in a reference memory and served as an estimate of the number of stimuli. Storage of pulse totals was subject to alteration in the total, thus giving rise to scalar variation in memory for a distribution of totals. Gallistel and Gelman (1992) extended this model by suggesting that the accumulator mechanism is innate and is to be found in both animals and people. Although humans had mapped verbal numerical symbols onto accumulator magnitudes, evidence could still be found for the accumulator process (Dehaene, 1997; Dehaene \& Akhavein, 1995). Thus, the finding that animals, children, and adults counting nonverbally show evidence of Weber's law and scalar variability in numerical studies supports the hypothesis that they share a common accumulator process.

In a second experiment performed by Whalen et al. (1999), people were shown a sequence of flashes at an aperiodic rate and were instructed not to count the flashes. Mean estimates of the number of flashes presented were close to the actual number, and variation in flash estimates increased in proportion to the mean estimate. The authors argued that the subjects could map bidirectionally, either from number symbol to magnitude (the keypress study) or from magnitude to number symbol (the flash-count study).

In this article, we present three experiments that are an extension of the flash-count study of nonverbal counting. In Whalen et al.'s (1999) experiment, subjects were simply told not to count verbally but could have done so. In the experiments presented here, subjects were required to perform a demanding cognitive task during item presentation that prevented them from counting the number of items presented. People were presented with sequences of simple geometric patterns and were asked to name properties of these patterns as they appeared and not to count the patterns. In Experiment 1, the subjects first saw a target number of patterns and then were asked for a verbal estimate of the number of patterns that had appeared or were asked to respond to a new set of patterns by pressing a space bar until they were sure that the number of patterns seen exceeded the target number. When the frequency of barpresses was plotted against the number of patterns shown, the peak of the curve provided a manual estimate of the target number. In Experiments 2 and 3, a matching-tosample procedure was used in which both verbal and manual estimates of the sample target number were obtained on each trial. The novel features of these experiments were (1) preventing the subjects from counting subvocally by requiring them to perform other cognitive tasks, (2) the measurement of nonverbal (manual) production of number by the peak procedure, (3) the measurement of verbal and manual estimates of the same target number on individual trials, and (4) a comparison of nonverbal number 
estimation when the subjects did or did not have to perform another number estimation task during pattern presentation (Experiments 2 and 3).

Several theoretical questions were addressed by these experiments. Will subjects accurately estimate the number of patterns shown when they are prevented from counting verbally? Will the distribution of estimates show scalar variation as the number of items estimated increases? The dual-mode model suggests that subjects should be providing estimates of a common magnitude when asked to give both verbal and manual responses on the same trial. Will error in verbal and manual estimates show positive correlations, indicating that they represent alternate indicators of the same underlying representation? Finally, the subjects were asked to name pattern shapes and colors in Experiments 1 and 2 but were required to name the color and to estimate the number of objects in patterns shown in Experiment 3. Will requiring subjects to perform another number estimation task affect their estimates of the number of patterns shown?

\section{EXPERIMENT 1}

\section{Method}

\section{Subjects and Apparatus}

Twenty-four undergraduate students from the University of Western Ontario (15 women and 9 men) served as subjects. Twelve students participated in partial fulfillment of the requirements of an introductory psychology course. Twelve other subjects were paid \$30 at the completion of the experiment. An IBM-compatible computer (486) presented stimuli on a color monitor. Response data were recorded through the keyboard. A custom program written in Microsoft VisualBasic 4.0 controlled presentation of stimuli and recorded responses.

\section{Procedure}

The subjects were tested in three separate sessions on 3 different days. Each session tested one of three target numbers $(8,16$, or 32$)$. The order in which the targets were tested was counterbalanced across participants.

Training (10 trials). Each subject was instructed to press the Enter key to begin the session. A purple circle appeared on screen and persisted for 1-3 sec (randomly varied) to identify the start of each trial. One second following the termination of this warning stimulus, individual patterns appeared in sequence until the target number was reached. Immediately after the target pattern, an end-oftrial (EOT) stimulus appeared, which was identical to the warning stimulus. Patterns were colored (red, green, blue, yellow, or white) geometric shapes (square, rectangle, circle, or ellipse) of approximately equal area. At the start of a session, the program compiled a large list of color-shape combinations by random selection (with replacement). Each pattern appeared on screen for $0.5 \mathrm{sec}$. The interitem interval varied randomly within the range of $0.3-2.4 \mathrm{sec}$. Intertrial intervals (ITIs) varied randomly between 3 and $5 \mathrm{sec}$.

The subjects were asked to say aloud the color and shape of each pattern (except warning and EOT stimuli) as soon as it appeared on screen on every trial. They were instructed to "get a feel" for how many patterns appeared on each trial and to avoid actively counting the patterns. Note that during training, each trial consisted of exactly the target number $(8,16$, or 32$)$ of patterns. On Trials $6-10$, the subjects were further instructed to begin to repeatedly press the space bar during each trial when he or she was confident that the target pattern would soon appear and to continue to press the space bar until the EOT appeared. For example, on a trial with 16 items, a sub- ject might begin pressing the space bar after the 13th or 14th item, indicating his or her awareness that the 16th item would soon appear. The subjects were told they should press the space bar at least five times per trial.

Testing. A testing phase of 30 trials followed the training phase. These trials differed from training trials in two respects. Fifteen trials were empty trials, in which the EOT stimulus was withheld and patterns continued to appear beyond the target number. The subjects were again instructed to begin tapping the space bar when they felt confident that the target number would soon appear and then to press the End key when they felt confident that the target number had been surpassed. This action terminated the trial and instigated an ITI.

Fifteen other trials (question trials) presented four questions on screen following the EOT stimulus. These questions required the subjects to estimate four properties of the set of patterns just seen: (1) the number of patterns belonging to a particular color category, (2) the number of patterns belonging to a particular shape category, (3) the duration of presentation of the set, and (4) the total number of patterns in the set. In the case of Questions 1 and 2, a color and shape were chosen at random. The first three questions were designed to distract the subjects from the true purpose of the experiment; only responses to Questions 3 and 4 were recorded.

The distribution of question and empty trials was arranged pseudorandomly, with the restriction that the same trial type could not be repeated more than three times in succession. No feedback regarding accuracy was provided to the subjects.

\section{Results}

Degrees of freedom for each repeated measures analysis of variance (ANOVA) were adjusted with the GreenhouseGeisser epsilon, following Kirk (1995). Alpha was set at .05 for all statistical tests. All post hoc tests were $t$ tests.

\section{Manual Response}

Data from empty trials were expressed as rates of responding in successive number bins, which corresponded to the intervals during which a geometric pattern was presented. Data were averaged over trials for each subject, and overall mean peak number curves were computed for each target number. Curves were smoothed (by averaging each point, except the first and the last, with its neighbors) and then were normalized to a common peak of 1.0 by dividing each point by the peak rate of space bar pressing (see Roberts \& Boisvert, 1998). Peak rates of barpressing on targets 8,16 , and 32 occurred at 9,17 , and 32 patterns, respectively (Figure 1, top panel).

The midpoint of each response run was computed on every trial as $S+[(E-S) / 2]$, where $S$ (start) indicates the number at which the response run began and $E$ (end) indicates the first number with no responses. The mean midpoints at targets 8,16 , and 32 were $8.29,16.40$, and 30.66 , respectively (Figure 2, top panel). A one-way ANOVA for repeated measures showed that these three means differed significantly $[F(2,28)=185.21]$. Subsequent $t$ tests indicated that each pair of means differed significantly $[t(23) \geq$ 5.35].

Scalar variability in manual estimates was analyzed by comparing COVs from mean midpoints at each target number. Mean COVs obtained at target 8 , target 16, and target 32 were $.14, .13$, and .15 , respectively; these values did not differ statistically $(F<1.0)$. 


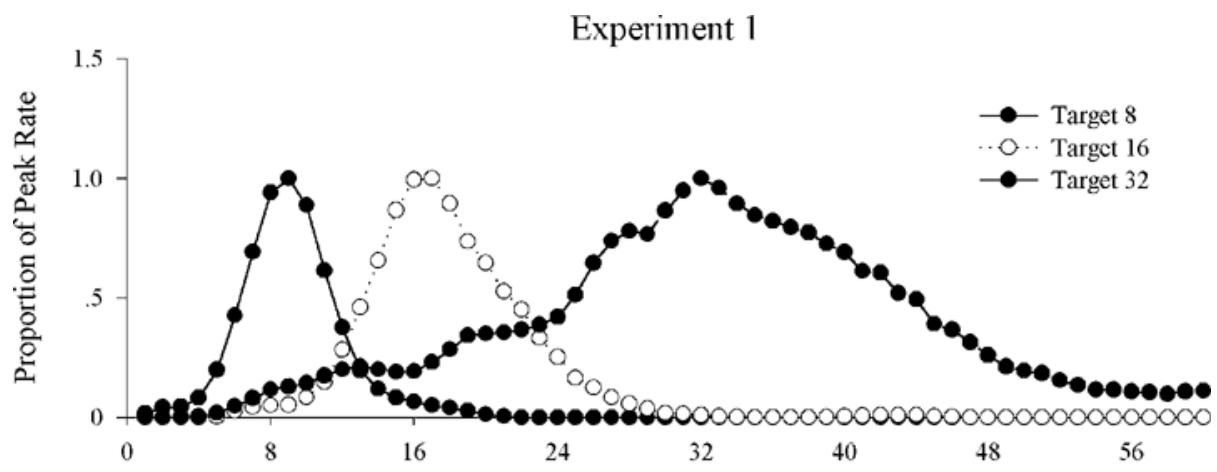

Experiment 2
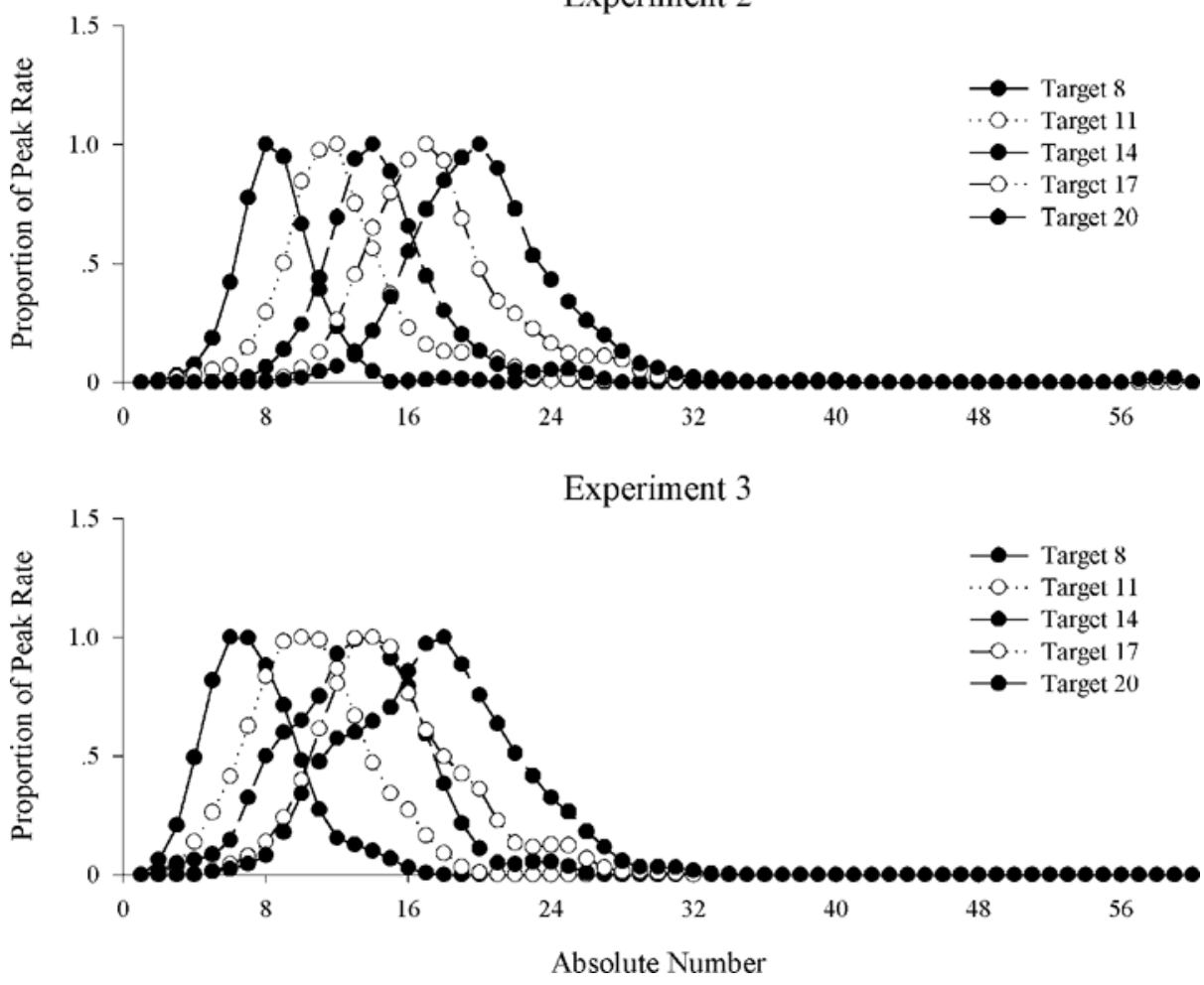

Figure 1. Peak number curves obtained from empty trials at target 8 , target 16, and target 32 schedules in Experiment 1 (top panel), and the matching phase of trials at targets 8, 11, 14, 17, and 20 in Experiment 2 (middle panel) and Experiment 3 (bottom panel). Curves have been smoothed and normalized (see the text) to plot proportion of peak rate of responding.

\section{Verbal Response}

Verbal estimates of each target were calculated for each subject from question trials. Mean verbal estimates of the targets 8,16 , and 32 schedules were $8.48,15.84$, and 28.39 , respectively (Figure 2, top panel). These estimates differed significantly from each other $[F(2,46)=244.54]$; all pairs of means differed significantly $[t(23) \geq 6.70]$. Paired samples $t$ tests revealed that mean verbal estimates and mean midpoint numbers at each target did not differ significantly $[t(23)<1.44]$.

The scalar property was also evident in verbal estimates of target size. Mean verbal COVs were $.09, .11$, and .11 at targets 8,16 , and 32 , respectively; these values were not significantly different $(F<1.0)$.

To investigate whether the subjects used target duration, rather than a counting process, correlations were computed between verbal estimates of target duration, actual duration, and verbal estimates of target number. A correlation was obtained for each subject on each pair of variables, and overall mean correlations were computed at each target number. Each mean was then tested against a value of zero. The mean correlation between verbal estimates of target duration and target number differed significantly from zero at all target numbers [target $8, M=.39, t(20)=$ 
4.9; target $16, M=.32, t(21)=4.08 ;$ target $32, M=.38$, $t(20)=4.9]$. The mean correlation between actual target duration and verbal estimates of it differed from 0 only at target $8[M=.2, t(23)=4.46$; target $16, M=.05, t(22)=$ .85 ; target $32, M=.09, t(22)=1.31]$. Most important, mean correlations between verbal estimates of target number and actual target duration did not differ from 0 at any target number [target $8, M=.07, t(17)=1.05$; target 16 , $M=.1, t(22)=1.77 ;$ target $32, M=.07, t(20)=1.03]$. The most likely explanation for the significant relationship between verbal estimates of target number and target duration is that it made little sense to the subjects to enter a large estimate of number and a low estimate of duration and vice versa. The absence of correlation between actual target duration and number estimate suggests that time was not used to estimate number.

\section{Discussion}

The subjects practiced on each of the three target number schedules showed peak responding on empty trials very near the target number. The spreads of peak number functions increased with the magnitude of the target number,

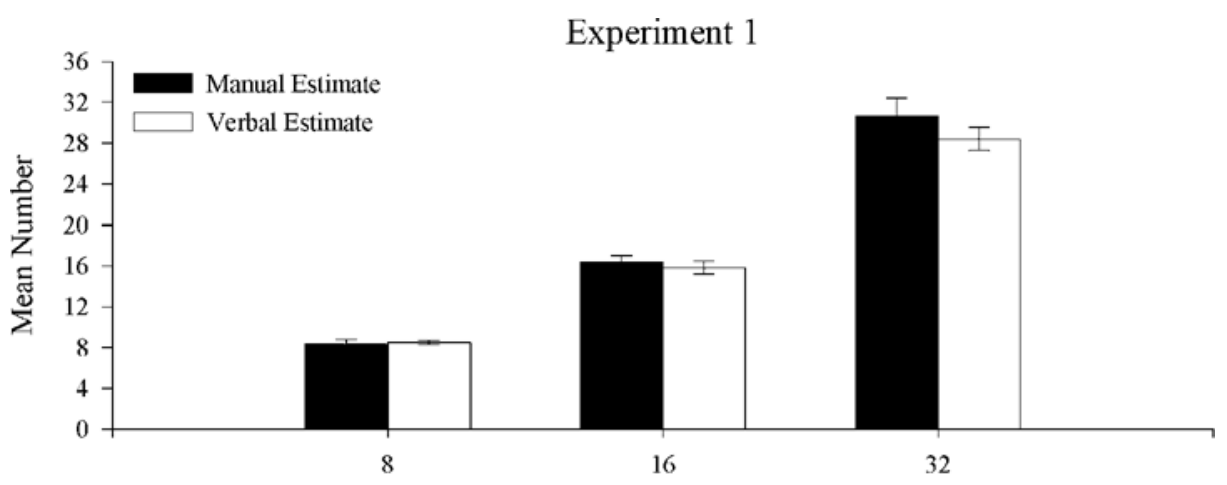

Experiment 2

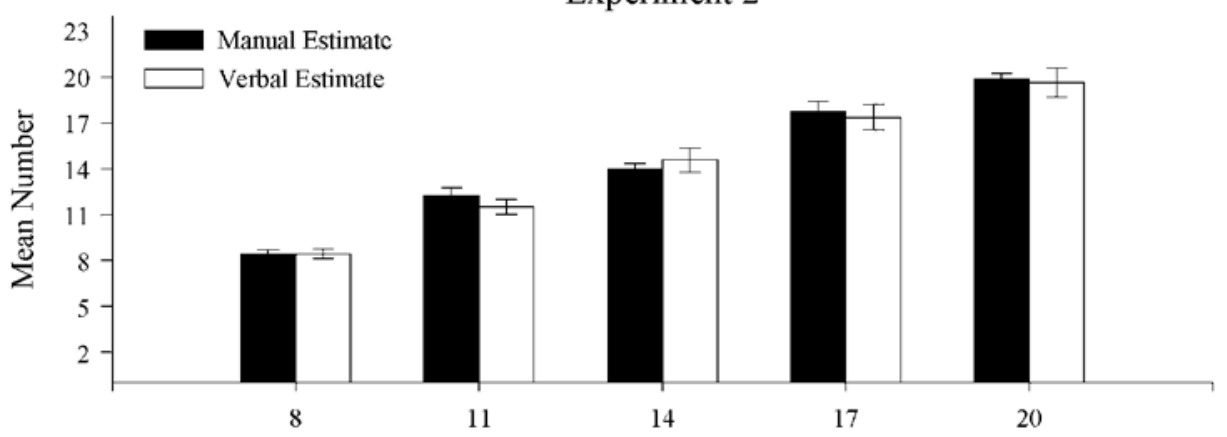

Experiment 3

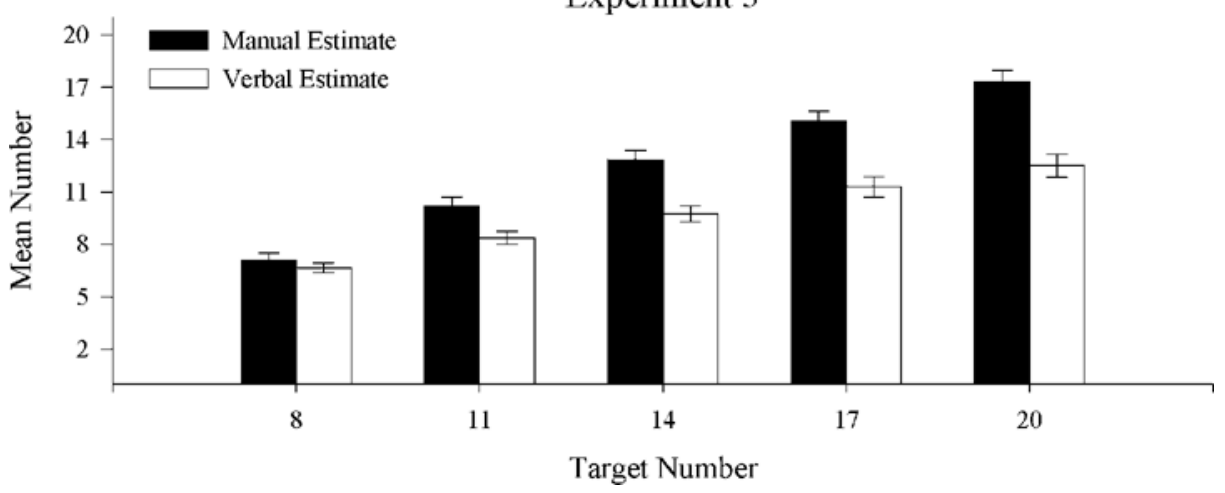

Figure 2. Mean ( $\pm S E$ ) verbal estimates (light bars) obtained from responses on individual question trials in Experiment 1 (top panel) and on the sample phase of Experiment 2 (middle panel) and Experiment 3 (bottom panel). Mean $( \pm S E$ ) manual estimates (dark bars) obtained from responses on individual empty trials in Experiment 1 (top panel) and on the matching phase of Experiment 2 (middle panel) and Experiment 3 (bottom panel) are also shown. 
suggesting the scalar property common in nonverbal counting (Roberts \& Boisvert, 1998; Whalen et al., 1999). Scalar variability in manual and verbal estimation was confirmed statistically, since COVs were invariant across target sizes. Finally, mean manual and verbal estimates of target size were approximately equally accurate. Analyses of the correlations between verbal estimates of target duration, actual duration, and target number suggested that the subjects' accurate estimates were based on a counting process, not a timing process.

Together, these results suggest that the distractor task successfully discouraged the subjects from verbally counting items. Furthermore, because COVs decrease as target size increases when subjects verbally count (Cordes et al., 2001), these data support the conclusion that the subjects were using a nonverbal counting process. These data suggest, therefore, that nonverbal estimates of target size were accessible to verbal report. If nonverbal and verbal estimates represent a common magnitude, the two should correlate positively. Experiment 2 tested this hypothesis, using a procedure that measured nonverbal and verbal estimates on the same trial.

\section{EXPERIMENT 2}

\section{Method}

\section{Subjects and Apparatus}

Twelve students ( 6 females and 6 males) from the University of Western Ontario served as subjects. None of these students had participated in Experiment 1. The same apparatus as that in Experiment 1 was used.

\section{Procedure}

Each subject was tested in two 1-h sessions. In each session, five target numbers $(8,11,14,17$, and 20) were tested over 35 trials ( 7 trials/number). The distribution of trials within a session was randomly determined, with the restriction that each target number appeared once in each 5-trial block. A sample phase preceded a matching phase on each trial. ITIs were the same as those in Experiment 1.

Sample phase. Following the warning stimulus, individual patterns appeared in sequence until the target was reached. All the properties of the patterns (color, shape, area, and interpattern intervals) were the same as those used in Experiment 1, except that each pattern now appeared for $0.35 \mathrm{sec}$. The subjects were instructed to refrain from actively counting the patterns but, rather, to try to "get a feel" for how many patterns appeared (excluding warning and EOT stimuli). Again, the subjects were required to identify verbally the color and shape of each pattern. Immediately following the last pattern in the set, the subjects were asked on screen, "How many patterns appeared in that set?" The subject's response ended the sample phase.

Matching phase. The matching phase began $1 \mathrm{sec}$ after the sample phase. Individual patterns appeared in sequence until four times the target number of patterns was reached or until the subject ended the trial with a keystroke. During this phase, the subjects were required to estimate manually the location of the target number within this set. That is, they were required to manually "match" the number representing the target number from the sample phase. Each subject was instructed to begin tapping the space bar when he or she was confident that the number of patterns corresponding to the target from the sample phase would soon appear and to terminate tapping and press the End key when confident that this number had been surpassed. The subjects were required to call aloud the color and shape name of each pattern in the set. All shapes, colors, and interitem intervals were randomly selected and, thus, were independent of those that made up the sample phase. An ITI that varied randomly between 3 and $5 \mathrm{sec}$ followed each trial.

\section{Results}

\section{Manual Response}

Peak number curves produced from response data (as in Experiment 1) are shown in Figure 1 (middle panel). The subjects accurately estimated the location of the target from the sample phase: Peak rates of pressing on targets 8 , $11,14,17$, and 20 samples occurred at $8,12,14,17$, and 20 , respectively.

The subjects' nonverbal estimates of target number were taken by computing the midpoint number from tapping responses, as in Experiment 1. Mean midpoints on targets 8, $11,14,17$, and 20 trials were $8.41,12.22,14.08,17.75$, and 19.81, respectively. These estimates did not differ from their corresponding targets $[t(11)<1.34]$, except in the case of target $11[t(11)=2.26]$. Mean COV, computed using midpoints, as in Experiment 1, did not differ significantly across target sizes $[.19, .22, .17, .17, .16 ; F(4,44)=$ 2.43].

\section{Verbal Response}

The subjects provided accurate verbal estimates of each target number at the end of sample presentation (Figure 2, middle panel). Mean estimates on targets $8,11,14,17$, and 20 trials were $8.43,11.52,14.58,17.38$, and 19.65 , respectively; none of these estimates differed significantly from its corresponding sample value $[t(11)<1.5]$. Mean COVs were constant across target sizes $(.12, .14, .14, .15$, $.14 ; F<1.0)$.

\section{Comparison of Verbal and Manual Estimates}

Difference scores were computed to investigate error in estimation. The target for each trial was subtracted from its verbal and manual estimates, and each difference then was divided by the target itself. These two quotients, the difference scores, reflect the direction and degree of error in estimating the target. Negative scores indicate underestimation, positive scores indicate overestimation, and zero scores indicate perfect estimation. These scores were correlated over all trials for each subject. Positive, significant correlations were obtained for all but 1 subject. The mean correlation coefficient $(M=.56)$ differed significantly from zero $[t(11)=7.17, p<.01]$.

\section{Discussion}

Experiment 2 provides additional evidence for the use of a nonverbal counting process and extends the results of Experiment 1 by showing positive correlations between manual and verbal estimates of target number. The pattern of results suggests that both estimates reflect a common magnitude. The subjects were prevented from verbal counting in these experiments by a cognitive distraction task, which involved naming the colors and shapes of items within the set. In Experiment 3, the distraction task involved verbally counting objects in each pattern and estimating the target 
number. Would performing a separate verbal counting task affect verbal and nonverbal estimates of target size?

\section{EXPERIMENT 3}

\section{Method}

\section{Subjects and Apparatus}

Ten students ( 9 females, 1 male) from the University of Western Ontario were paid \$25 for participating. None of these students had participated in the previous experiments. The same apparatus as that in Experiments 1 and 2 was used.

\section{Procedure}

The procedure used here was identical to that in Experiment 2, with two exceptions. First, each pattern now comprised between 1 and 10 identical colored shapes. The number, type, and color of the shapes making up each pattern were randomly determined. These features of a pattern were also randomized across patterns in each target set. The subjects were required to say aloud the number of shapes in each pattern and their color (e.g., "eight red") and then to verbally estimate the number of patterns that had been presented (i.e., the target number). Second, each pattern now appeared for $0.5 \mathrm{sec}$. All other properties of the trials and the instructions were the same as those in Experiment 2.

\section{Results}

\section{Manual Response}

Peak numbercurves are shown in Figure 1 (bottom panel). Peak rates of pressing on targets $8,11,14,17$, and 20 trials occurred at $6,10,14,14$, and 18 , respectively. Mean midpoints at the five target sizes were $7.08,10.2,12.83$, 15.06 , and 17.3, respectively. Estimates at targets 8 and 11 did not differ significantly from their corresponding target $[t(9)<2.18]$. Estimates at targets 14,17 , and 20 were significantly below target $[t(9)>2.23]$. Mean COVs were constant across targets $[.26, .25, .24, .22, .21 ; F(4,36)=$ 1.79]. Although these means are strictly descending, no individual subject showed this pattern across all the targets.

\section{Verbal Response}

The subjects' verbal estimates of all the sample numbers underestimated the target (Figure 2, bottom panel). Mean verbal estimates on targets $8,11,14,17$, and 20 trials were $6.66,8.37,9.75,11.30$, and 12.51 , respectively. Each of these estimates was significantly below its corresponding target $[t(9)<4.56]$. Despite this poor performance, variability in these estimates increased in proportion to the mean estimates: Mean COVs were constant across target sizes $(.14, .15, .17, .16, .16 ; F<1.0)$.

\section{Comparison of Verbal and Manual Estimates}

Difference scores representing underestimation and overestimation were computed as in Experiment 2. Positive, significant correlations were obtained between verbal and manual scores for all the subjects. The mean correlation coefficient $(M=.54)$ differed significantly from zero $[t(9)=11.51]$.

\section{GENERAL DISCUSSION}

Adult humans estimated target numbers in peak number procedures borrowed from the animal literature. The subjects were discouraged from verbal counting by instructions not to count and by cognitive tasks in which they named features of the patterns to be enumerated. Manual and verbal estimates were accurate for numerosities up to about 30 . The variability in these estimates increased in proportion to target magnitude. The scalar property evident in these data supports the conclusion that the subjects, in fact, did not verbally count patterns. Using a different procedure from that used here, Cordes et al. (2001) showed that verbal counting can be distinguished from nonverbal counting by unique patterns of variability. A constant COV characterized responses to a target stimulus by subjects distracted from verbal counting, but subjects asked to verbally count their responses made fewer errors at higher target values. Our results are qualitatively similar to the animal data (e.g., Roberts \& Boisvert, 1998) and other work with adult humans (Cordes et al., 2001; Whalen et al., 1999), despite the use of different procedures, and provide further support for the accumulator counting model proposed by Meck and Church (1983).

One version of Meck and Church's (1983) model suggests that verbal and nonverbal responses estimate a common magnitude (Gallistel \& Gelman, 1992). Similar accuracy in verbal and manual estimates and positive correlations between these estimates support this proposition. If a common magnitude underlies both responses, verbal and nonverbal estimates should correlate positively, because they err by roughly the same degree. In Experiments 2 and 3, positive correlations were obtained between verbal and manual estimates of the same target presentation. Verbal estimates corresponded closely to manual estimates when the distraction task involved naming pattern colors and shapes (Experiment 2) but fell short of both target numbers and manual estimates when the distraction task involved giving a verbal estimate of the number of shapes (Experiment 3). One possible explanation for this pattern of results is that repeatedly estimating the small numerosities (i.e., 1 to 10 shapes) shown as patterns biased verbal estimates of number of patterns toward small numbers. However, there was evidence that manual responses also underestimated the higher target numbers of patterns $(14,17$, and 20). This systematic underestimation of the target number of patterns suggests the interesting possibility that requiring the subjects to estimate the number of shapes shown on each pattern may have interfered with their ability to count the number of patterns. Because each pattern was shown for only $0.5 \mathrm{sec}$ and each pattern contained 1-10 shapes, the cognitive activity performed during pattern presentation may be viewed as subitizing. Although there is debate concerning the basis of subitizing, Gallistel and Gelman argued that a nonverbal accumulator process is responsible for estimation of briefly seen quantities. During pattern presentation in Experiment 3, therefore, the subjects may have been performing two accumulator processes, one to keep track of the total number of patterns and the other to estimate the number of shapes shown on each pattern. If the latter process interfered with the former process of accumulating number of patterns, the magnitude available at the end of the sample 
phase would underestimate the target number. Manual underestimates may have been caused by this lower magnitude, and the even lower verbal estimates may have arisen from an additional bias to give low verbal estimates based on the restricted range (1-10) of shapes subjects responded to verbally during pattern presentation. The positive correlations between verbal and manual estimates indicate that both responses were estimating a common representation, but the underestimation seen in Experiment 3 raises the interesting possibility that interactions between multiple accumulators can be studied in nonverbal counting experiments.

\section{REFERENCES}

Clearfield, M. W., \& Mix, K. S. (1999). Number versus contour length in infants' discrimination of small visual sets. Psychological Science, 10, 408-411.

Cordes, S., Gelman, R, Gallistel, C. R., \& Whalen, J. (2001). Variability signatures distinguish verbal from nonverbal counting for both large and small numbers. Psychonomic Bulletin \& Review, 8, 698707.

Dehaene, S. (1997). The number sense: How the mind creates mathematics. New York: Oxford University Press.

Dehaene, S., \& Akhavein, R. (1995). Attention, automaticity, and levels of representation in number processing. Journal of Experimental Psychology: Learning, Memory, \& Cognition, 21, 314-326.

Feigenson, L., Carey, S., \& Hauser, M. (2002). The representations underlying infants' choice of more: Object files versus analog magnitudes. Psychological Science, 13, 150-156.

Gallistel, C. R., \& Gelman, R. (1992). Preverbal and verbal counting and computation. Cognition, 44, 43-74.

Huntley-Fenner, G. (2001). Children's understanding of number is similar to adults' and rats': Numerical estimation by $5-7$-year-olds. Cognition, 78, B27-B40.

Huntley-Fenner, G., \& Cannon, E. (2000). Preschoolers' magnitude comparisons are mediated by a preverbal analog mechanism. Psychological Science, 11, 147-152.

KIRK, R. E. (1995). Experimental design: Procedures for the behavioral sciences (3rd ed.). Pacific Grove, CA: Brooks/Cole.

Mechner, F. (1958). Probability relations within response sequences under ratio reinforcement. Journal of the Experimental Analysis of Behavior, 1, 109-121.

Meck, W. H., \& Church, R. M. (1983). A mode control model of count- ing and timing processes. Journal of Experimental Psychology: Animal Behavior Processes, 9, 320-334.

Platt, J. R., \& Johnson, D. M. (1971). Localization of position within a homogeneous behavior chain: Effects of error contingencies. Learning \& Motivation, 2, 386-414.

RoBerts, W. A. (1998). Principles of animal cognition. Boston: McGrawHill.

Roberts, W. A. (2002). Mechanisms of "counting" in animals. In S. B. Fountain, M. D. Bunsey, J. H. Danks, \& M. K. McBeath (Eds.), Animal cognition and sequential behavior (pp. 153-174). Boston: Kluwer.

Roberts, W. A., \& Boisvert, M. J. (1998). Using the peak procedure to measure timing and counting processes in pigeons. Journal of Experimental Psychology: Animal Behavior Processes, 24, 416-430.

Roberts, W. A., Coughlin, R, \& Roberts, S. (2000). Pigeons flexibly time or count on cue. Psychological Science, 11, 218-222.

Roberts, W. A., Roberts, S., \& KIt, K. A. (2002). Pigeons presented with sequences of light flashes use behavior to count but not to time. Journal of Experimental Psychology: Animal Behavior Processes, 28, 137-150.

Simon, T. J. (1997). Reconceptualizing the origins of number knowledge: A "non-numerical” account. Cognitive Development, 12, 349-372.

Starkey, P., \& Cooper, R. G., JR. (1980). Perception of numbers by human infants. Science, 210, 1033-1035.

Starkey, P., Spelke, E. S., \& Gelman, R. (1990). Numerical abstraction by human infants. Cognition, 36, 97-127.

Strauss, M. S., \& CurTis, L. E. (1981). Infant perception of numerosity. Child Development, 52, 1146-1152.

VAn Loosbroek, E., \& Smitsman, A. W. (1990). Visual perception of numerosity in infancy. Developmental Psychology, 26, 916-922.

Whalen, J., Gallistel, C. R., \& Gelman, R. (1999). Nonverbal counting in humans: The psychophysics of number representation. Psychological Science, 10, 130-137.

WynN, K. (1992). Addition and subtraction by human infants. Nature, 358, 749-750.

WYNN, K. (1995). Origins of numerical knowledge. Mathematical Cognition, 1, 35-60.

WYNN, K. (1996). Infants' individuation and enumeration of actions. Psychological Science, 7, 164-169.

Wynn, K. (1997). Competence models of numerical development. Cognitive Development, 12, 333-339.

Xu, F., \& SPELKe, E. S. (2000). Large number discrimination in 6-monthold infants. Cognition, 74, B1-B11.

(Manuscript received October 2, 2001; revision accepted for publication July 17, 2002.) 